\title{
The Interplay of Intraband and Interband Polarization in the Ultrafast Response of Biased Semiconductor Superlattices
}

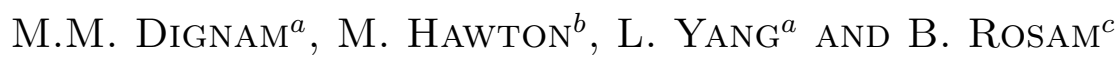 \\ ${ }^{a}$ Department of Physics, Queen's University \\ Kingston, ON, K7L 3N5, Canada \\ ${ }^{b}$ Department of Physics, Lakehead University \\ Thunder Bay, ON, P7B 5E1, Canada
}

${ }^{c}$ Institut für Angewandte Photophysik, Technische Universität Dresden

01062 Dresden, Germany

\begin{abstract}
We present the results of a new excitonic formalism for the treatment of ultrafast dynamics in asymmetric semiconductor multiple quantum well structures. The method is infinite order in the optical field, with truncation of the infinite hierarchy of dynamical equations being accomplished via a factorization of six-particle correlation functions into a product of twoand four-particle ones. We use this formalism to calculate the $\mathrm{THz}$ emission and degenerate four-wave mixing signals from biased semiconductor superlattices under different excitation conditions. We present a number of density-dependent effects that demonstrate the central role that the intraband polarization plays in determining and modifying the nonlinear ultrafast response of the system.
\end{abstract}

PACS numbers: 78.47.+p, 42.65.Re, 78.67.-n

\section{Introduction}

There has been considerable interest in recent years in the development of a clear theoretical understanding and treatment of the nonlinear optical response of semiconductor nanostructures to ultrashort optical pulses [1-7]. One common approach to this problem has been to expand the response in powers of the optical field $[2,4,5-7]$. This approach has been very successful for the treatment of many different nonlinear effects. However, there are many effects such as Rabi flopping that cannot be treated perturbatively. A method that is infinite order in the optical field is to employ the semiconductor Bloch equations (SBEs) in the Hartree-Fock approximation. This approach has been very successful in describing a number of 
phenomena such as the dynamic Stark effect. However, it has been shown $[5,8]$ that it is not suited to structures in which excitonic effects are strong, such as biased semiconductor superlattices (BSSLs), as it underestimates the electron-hole correlations in the intraband response. In this work, we employ the excitonic Bloch equations of Hawton and Dignam [3] to calculate the intraband and interband response of a BSSL to ultrashort optical pulses. These equations are infinite order in the optical field and retain the important electron-hole correlations within an exciton.

\section{Results and discussion}

The dynamical behavior of electrons and holes in BSSLs have been investigated both theoretically $[1-7]$ and experimentally [7-10] by a large number of authors. The basic intraband dynamics can be understood in terms of the stationary states of non-interacting electrons in the superlattice potential in the presence of a uniform, along-axis static electric field, $F$. As has been well documented, these states have energies given by $E_{p}=E_{0}+e F p d$, where $d$ is the superlattice period, and $p$ is an integer. This forms the so-called Wannier-Stark ladder (WSL). The intraband dynamics can be simply understood in terms of the evolution of the time-dependent wavepacket formed from a superposition of these stationary states. Within a one-band approximation, this wavepacket undergoes periodic oscillations at the so-called Bloch frequency, $\omega_{\mathrm{B}}=e F d / \hbar$. This motion is known as Bloch oscillations (BOs).

This simple picture is modified when the electron-hole Coulomb interactions - i.e. excitonic effects - are taken into account $[2,3,6,7]$. These effects are particularly strong for low to moderate electric fields where the WSL energy spacing is comparable to the binding energy of the $1 s$ excitons. One of the key effects is that not only is the BO frequency modified, but many different BO frequencies arise, due to the different spacings between the excitonic WSL states. It is thus crucial in this system that any theoretical approach treat correctly the electronhole correlation effects within each exciton. It is for this reason that we employ an excitonic basis to treat the dynamics.

We consider a BSSL that is excited via an optical field given by $\boldsymbol{E}^{\mathrm{opt}}(\boldsymbol{R}, t)=$ $\sum_{\boldsymbol{K}} \boldsymbol{E}_{\boldsymbol{K}}^{\mathrm{opt}}(t)$, where for one-pulse THz experiments, $\boldsymbol{K}= \pm \boldsymbol{\kappa}_{1}$, while for two-pulse degenerate four-wave mixing (DFWM) experiments, the sum is over $\pm \boldsymbol{\kappa}_{1}$ and $\pm \boldsymbol{\kappa}_{2}$, where $\boldsymbol{\kappa}_{1}$ and $\boldsymbol{\kappa}_{2}$ are the wave vectors of the incident pulses. The Hamiltonian that we use to describe the system is given by $[2,3]$ :

$$
\begin{aligned}
H= & \sum_{\mu ; \boldsymbol{K}} \hbar \omega_{\mu} B_{\mu, \boldsymbol{K}}^{\dagger} B_{\mu, \boldsymbol{K}}-V \sum_{\boldsymbol{K}}\left(\boldsymbol{E}_{-\boldsymbol{K}}^{\mathrm{opt}} \cdot \boldsymbol{P}_{\boldsymbol{K}}^{\mathrm{inter}}+\text { h.c. }\right) \\
& +V \sum_{\boldsymbol{k}}\left[\frac{1}{2 \epsilon} \boldsymbol{P}_{-\boldsymbol{k}}^{\mathrm{intra}}-\boldsymbol{E}_{\mathrm{ext}}^{\mathrm{THz}} \delta_{\boldsymbol{k}, 0}\right] \cdot \boldsymbol{P}_{\boldsymbol{k}}^{\mathrm{intra}},
\end{aligned}
$$


where $V$ is the system volume, $\hbar \omega_{\mu}$ is the energy and $B_{\mu, \boldsymbol{K}}^{\dagger}$ is the creation operator of the exciton (in the dc field) with internal quantum number, $\mu$, and center of mass wave-vector, $\boldsymbol{K}$. The external $\mathrm{THz}$ field (if present) is assumed to be spatially uniform over the structure and is given by $\boldsymbol{E}_{\text {ext }}^{\mathrm{THz}}(t)$. The interband polarization with wave vector $\boldsymbol{K}$ is given by $\boldsymbol{P}_{\boldsymbol{K}}^{\text {inter }}=\frac{1}{V} \sum_{\mu}\left(\boldsymbol{M}_{\mu} B_{\mu,-\boldsymbol{K}}^{\dagger}+\boldsymbol{M}_{\mu}^{*} B_{\mu, \boldsymbol{K}}\right)$, where $M_{\mu}$ is the excitonic interband dipole matrix element. Finally, the intraband polarization with wave vector $\boldsymbol{k}$ is $\boldsymbol{P}_{\boldsymbol{k}}^{\text {intra }}=\frac{1}{V} \sum_{\mu, \nu} \sum_{\boldsymbol{K}} \boldsymbol{G}_{\mu, \nu} B_{\mu, \boldsymbol{K}}^{\dagger} B_{\nu, \boldsymbol{k}+\boldsymbol{K}}$, where $\boldsymbol{G}_{\mu, \nu}$ is the intraband dipole matrix element between two excitonic states [2]. For DFWM, $\boldsymbol{K}$ in the third sum in Eq. (1) runs over the values, $\boldsymbol{K}_{m}=m\left(\boldsymbol{\kappa}_{1}-\boldsymbol{\kappa}_{2}\right)$, where $m$ is an integer. This gives the wave vectors of all of the polarization gratings created via the interfering excitonic waves. The first sum in Eq. (1) runs over wave vectors, $\boldsymbol{K}_{n}=(n+1) \boldsymbol{\kappa}_{1}-n \boldsymbol{\kappa}_{2}$, where $n$ is an integer. This gives the wave vectors of all of the excitons that are scattered out of the original directions via the intraband polarization gratings. For single beam experiments, these equations still hold with $\boldsymbol{\kappa}_{2}=\boldsymbol{\kappa}_{1}$. In deriving this Hamiltonian, we have neglected the exchange interaction (which is valid for the densities considered) and have approximated the exciton-exciton interaction via a dipole-dipole interaction [2].

We use the Heisenberg equations to determine operator dynamics. This creates an infinite hierarchy of dynamic equations. We truncate the hierarchy by factoring the three-exciton (six-particle) correlation functions into one- and two-exciton correlation functions. Thus, for example $\left\langle B_{\mu, \boldsymbol{K}_{1}}^{\dagger} B_{\nu, \boldsymbol{K}_{2}}^{\dagger} B_{\beta, \boldsymbol{K}_{3}}\right\rangle \rightarrow$ $\left\langle B_{\mu, \boldsymbol{K}_{1}}^{\dagger} B_{\beta, \boldsymbol{K}_{3}}\right\rangle\left\langle B_{\nu, \boldsymbol{K}_{2}}^{\dagger}\right\rangle$. Note that this factorization is very different than that used in the SBE's, as it retains the intra-excitonic electron-hole correlations and the long range exciton-exciton correlations. We have shown that this factorization is accurate for the calculation of DFWM signals to third order in BSSL's [2]. Our method is similar to the approach of Axt and Mukamel [1] except that our use of the dipole approximation for the exciton-exciton interaction makes the equations more computationally feasible for complicated systems such as BSSLs. We account for dephasing and decoherence phenomenologically via the interband and intraband dephasing times, $T_{\mu}$ and $T_{\mu \nu}$, respectively. The dynamical equation for $\left\langle B_{\mu, \boldsymbol{K}_{n}}^{\dagger}\right\rangle$ thus becomes

$$
\begin{gathered}
\mathrm{i} \hbar \frac{\mathrm{d}\left\langle B_{\mu, \boldsymbol{K}_{n}}^{\dagger}\right\rangle}{\mathrm{d} t}=-\hbar\left(\omega_{\mu}+\frac{\mathrm{i}}{T_{\mu}}\right)\left\langle B_{\mu, \boldsymbol{K}_{n}}^{\dagger}\right\rangle+\boldsymbol{E}_{-\boldsymbol{K}_{n}}^{\mathrm{opt}} \cdot \boldsymbol{M}_{\mu}^{*} \\
\quad+\sum_{\nu ; \boldsymbol{K}_{m}} \boldsymbol{E}_{\boldsymbol{K}_{n}-\boldsymbol{K}_{m}}^{\mathrm{intra} *} \cdot \boldsymbol{G}_{\nu, \mu}\left\langle B_{\nu, \boldsymbol{K}_{m}}^{\dagger}\right\rangle,
\end{gathered}
$$

where $\boldsymbol{E}_{-\boldsymbol{K}}^{\text {intra }} \equiv-\left\langle\boldsymbol{P}_{\boldsymbol{K}}^{\text {intra }}\right\rangle / \epsilon+\boldsymbol{E}_{\text {ext }}^{\mathrm{THz}} \delta_{\boldsymbol{K}, 0}$ is the total self-generated near-field intraband field with the wave vector $\boldsymbol{K}$, where $\epsilon$ is the permittivity. There is a corresponding equation for the intraband correlation functions $\left\langle B_{\mu, \boldsymbol{K}_{n}}^{\dagger} B_{\nu, \boldsymbol{K}_{m}}\right\rangle$, 
which closes the set of equations. In deriving these equations, we have neglected the phase-space filling (PSF) terms associated with the fact that the excitons are not perfect bosons. The neglect of PSF terms is valid for this system for the densities considered in this paper. However, at higher densities, where the exciton Bohr radius becomes comparable to the inter-excitonic spacing PSF effects can become important. In these cases, these equations can be generalized to account for these effects [2].

The physics of the DFWM signal can be understood in a simple physical way from Eq. (2). The grating consisting of the intraband internal field with wave vector, $\boldsymbol{K}=\boldsymbol{\kappa}_{2}-\boldsymbol{\kappa}_{1}$, scatters the excitons with wave vector $\boldsymbol{\kappa}_{2}$ into the DFWM direction, $2 \boldsymbol{\kappa}_{2}-\boldsymbol{\kappa}_{1}$. Higher-order gratings scatter the excitons into different directions and perhaps back into the $2 \kappa_{2}-\kappa_{1}$ direction again. Of particular importance for response beyond third order is the internal intraband field with $\boldsymbol{K}=0$. This field interacts with the excitons in exactly the same way as does an external THz field: the dc component of this field renormalizes the excitonic energy, $\hbar \omega_{\mu}$, while its $\mathrm{THz}$ component dynamically couples levels with different $\mu$ [6]. As we shall see, it is this field that is largely responsible for the peak oscillations seen in spectrally-resolved DFWM signals and for the density-dependence of $\mathrm{THz}$ signals in single-pulse experiments.

We now present calculated results for DFWM and $\mathrm{THz}$ emission for the GaAs $/ \mathrm{Ga}_{0.7} \mathrm{Al}_{0.3}$ As superlattice used in recent experiments $[9,10,7]$ : the well widths and barrier widths are $67 \AA$ and $17 \AA$, respectively, the applied dc electric field is either $12.5 \mathrm{kV} / \mathrm{cm}$ (for THz results) or $15 \mathrm{kV} / \mathrm{cm}$ (for DFWM results), and there is no external THz field. The calculated linear absorption spectrum due to heavy-holes excitons for the two different dc fields is shown in Fig. 1. The spectra are dominated by heavy-hole $1 s$ excitonic states, which are labelled by the single internal quantum number, $\mu$, which describes the $1 s$ exciton in which the average along-axis electron-hole separation is approximately $\mu d[2]$. Thus, for the $1 s$ excitons, $\mu$ plays the role that $n$ does for the single-particle WSL states except that the excitonic WSL levels are not equally spaced. The dephasing times used in the dynamics calculations are taken to be $T_{\text {inter }}=T_{\mu}=1.0 \mathrm{ps}$ and $T_{\text {intra }}=$ $T_{\mu \nu}=1.5 \mathrm{ps}$ (similar to those observed in Ref. [8]), while the excitonic population decay time, $T_{\mu \mu}$, is taken to be infinite.

We begin with the results for the THz radiation emitted when $F=$ $12.5 \mathrm{kV} / \mathrm{cm}$ and the system is excited by a single Gaussian optical pulse with a spectral width (FWHM) of $21 \mathrm{meV}$ centered at $\omega_{\mathrm{c}}=\omega_{-1}$ (see Fig. 1). We include the excited in-plane states in addition to the dominant $1 \mathrm{~s}$ excitonic states. The states are calculated using the method given in Ref. [7]. In Fig. 2a, we plot the calculated uniform internal intraband field, $\boldsymbol{E}_{0}^{\text {intra }}$, as a function of time for several different pulse intensities (and hence different final carrier densities, $\rho$ ). Note that at all densities there is a sudden increase in the magnitude of the internal field when the pulse arrives. This field is due to the permanent dipole moments of the 


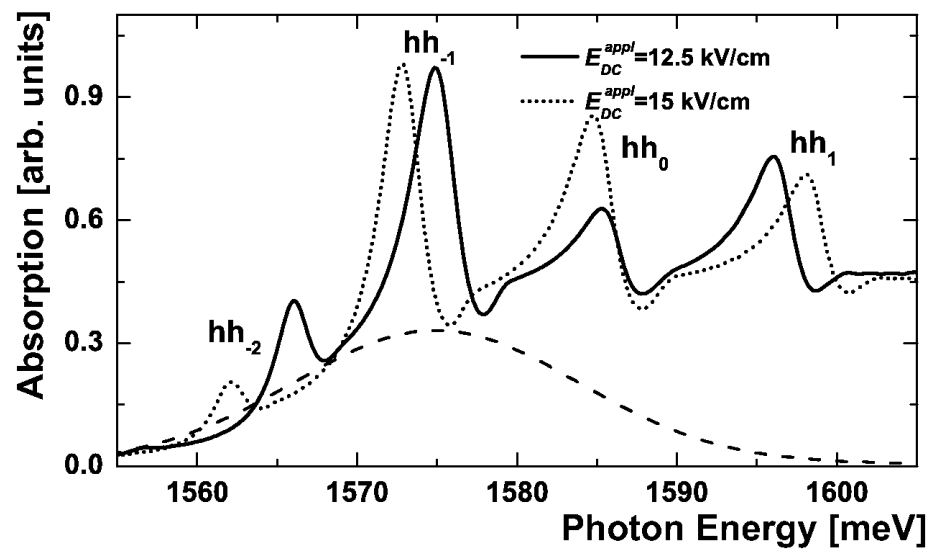

Fig. 1. The calculated absorption spectra of the BSSL in dc fields of $12.5 \mathrm{kV} / \mathrm{cm}$ and $15 \mathrm{kV} / \mathrm{cm}$. The power spectrum of the exciting optical pulse used for the $\mathrm{THz}$ results is also shown (broken line).

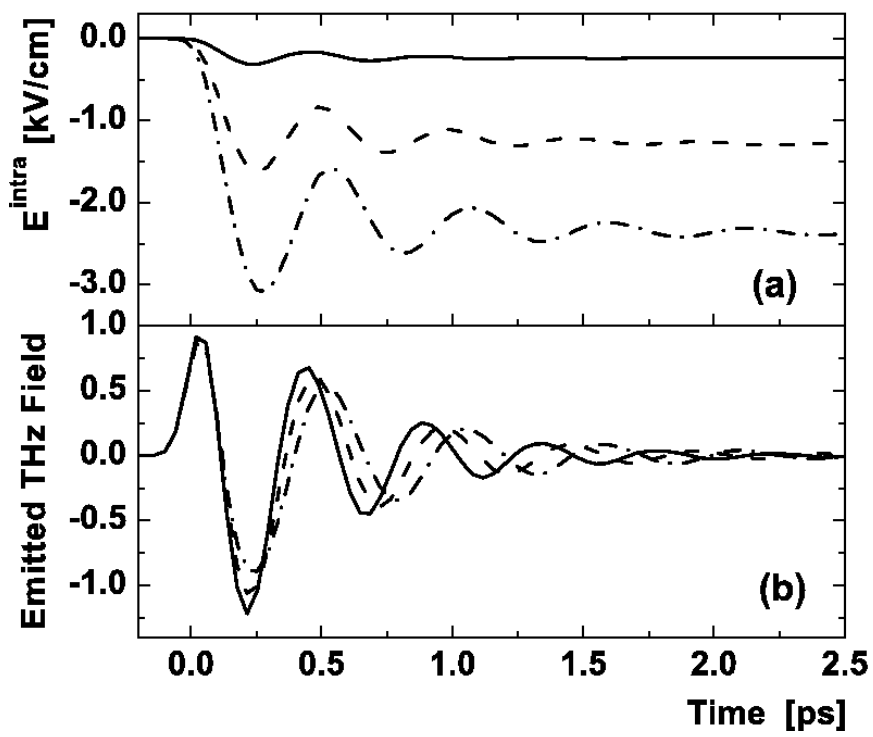

Fig. 2. The calculated intraband response to the Gaussian pulse for areal densities of $\rho=9.0 \times 10^{8} \mathrm{~cm}^{-2}$ (solid), $\rho=4.6 \times 10^{9} \mathrm{~cm}^{-2}$ (dash), and $\rho=8.2 \times 10^{9} \mathrm{~cm}^{-2}$ (dashdot). The internal intraband field, $E_{0}^{\text {intra }}(t)$, is shown as a function of time in (a), while the emitted $\mathrm{THz}$ field is shown as a function of time in (b). The inset shows the power spectra of the emitted $\mathrm{THz}$ fields.

excitons generated via the pulse, which for the $1 s$ excitonic WSL states is given by $-e \mu d$ as discussed above. Thus, since we are generating primarily excitons with $\mu<0$, this dc component of the intraband polarization is positive and the result- 
ing dc component of the generated intraband field, $\boldsymbol{E}_{0}^{\text {intra }}$, is less than zero, as observed. In addition to the sudden creation of a dc component to the intraband field, there is also a $\mathrm{THz}$ component arising from the Bloch-oscillating excitonic wavepackets.

In Fig. 2b, we plot the radiated $\mathrm{THz}$ field, calculated from the second derivative of $\boldsymbol{P}_{0}^{\text {intra }}(t)$. The oscillations in the field arise both from the sudden initial dipole and from the BOs, with the response at the later times being dominated by the BOs. As can be seen, the frequency of the oscillations is strongly dependent on the density, $\rho$. This density dependence is due to the fact that the dc component of $\boldsymbol{E}_{0}^{\text {intra }}(t)$ serves to oppose the applied dc field, leading to a net dc field that is less than the applied field. As the internal fields can be as large as $2.4 \mathrm{kV} / \mathrm{cm}$, this is a sizeable effect. Thus, we observe a red-shift in the $\mathrm{THz}$ frequency with increasing density.

We now turn to the results of DFWM calculations. The applied de field is $F=15 \mathrm{kV} / \mathrm{cm}$, and the exciting pulse is Gaussian with the same spectral FWHM of $21 \mathrm{meV}$ as shown in Fig. 1. In these calculations, only the $1 s$ heavy-hole excitons are included. The neglect of excited in-plane excitonic states has been shown to be justified for central laser frequencies well below the $\mu=0$ WSL frequency, as is the case here [7]. The calculations are performed by including excitonic states with the center of mass wave vectors, $\boldsymbol{K}_{n}=(n+1) \boldsymbol{\kappa}_{1}-n \boldsymbol{\kappa}_{2}$ for $n=-n_{0}, \ldots, n_{0}-1$, where $n_{0}$ is chosen so as to achieve convergence. In practice, we find that for the densities used here, convergence is reached for $n_{0}=6$.

In Fig. 3 we plot the spectrally-resolved DFWM (SR-DFWM) signal for a sequence of delay times for a density, $\rho=9.3 \times 10^{9} \mathrm{~cm}^{-2}$, with the pulse centered at $\omega_{\mathrm{c}}=\omega_{0}-2.27 \omega_{\mathrm{B}}$ (i.e., close to the $-2 \mathrm{WSL}$ state). The spectral peaks are associated with different excitonic states (as indicated in the figure). The peaks do not occur precisely at the single-exciton energies, $\omega_{\mu}$, due to the polarization-induced reduction of the applied dc field discussed above. More importantly, note that the peak positions depend significantly on the time delay, $\tau_{21}$.

To see the peak oscillations more clearly, we plot in Fig. 4 the energy of the $\mu=-1$ peak relative to $\hbar \omega_{-1}$ as a function of $\tau_{21}$ for $\rho=6.4 \times 10^{9} \mathrm{~cm}^{-2}$ for different central laser positions. As can be seen, and in agreement with recent experiments $[9,10]$, the peak's energy oscillates as a function of $\tau_{21}$ with period given approximately by $\tau_{\mathrm{B}} \equiv 2 \pi / \omega_{\mathrm{B}}$. The oscillation amplitude decreases as the central laser frequency approaches $\omega_{0}$ since the motion becomes more like a breathing mode the closer you come to the zero WSL state [9].

The peak oscillations can be largely understood as arising from quantum interference between multiple paths from the ground state to the excitonic state, $\left(\mu, 2 \boldsymbol{\kappa}_{2}-\boldsymbol{\kappa}_{1}\right)$. This can be seen through a simplified perturbative treatment illustrated in the inset to Fig. 3. In the left-hand path, an exciton with wave vector $\boldsymbol{\kappa}_{2}$ and energy $\hbar \omega_{\text {op }}$ is created by the second optical pulse; it then scatters off the $\boldsymbol{\kappa}_{2}-\boldsymbol{\kappa}_{1}$ grating into $2 \boldsymbol{\kappa}_{2}-\boldsymbol{\kappa}_{1}$, absorbing a photon of energy $\hbar \omega_{\text {intra }}^{G} \simeq 0, \pm \hbar \omega_{\mathrm{B}}$. In 


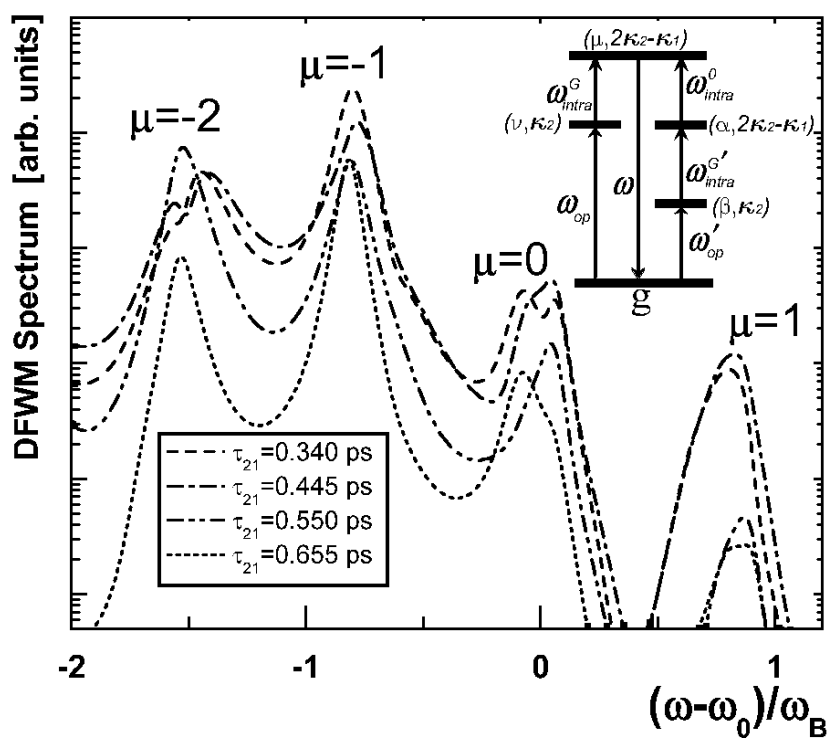

Fig. 3. The SR-DFWM intensity versus frequency for $\omega_{\mathrm{c}}=\omega_{0}-2.27 \omega_{\mathrm{B}}$ and $\rho=$ $9.3 \times 10^{9} \mathrm{~cm}^{-2}$ for a sequence of delay times. The inset shows the diagram for the quantum interference described in the text.

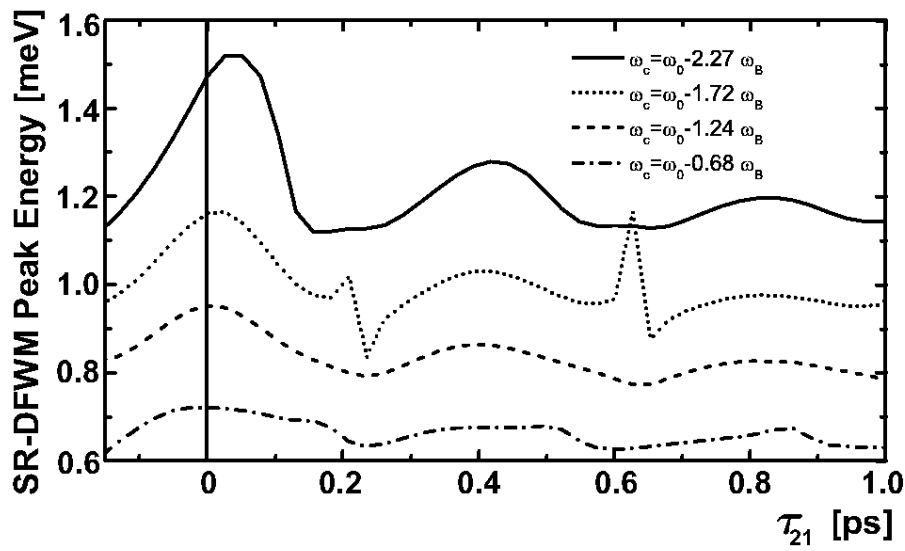

Fig. 4. The $\mu=-1$ SR-DFWM peak energy relative to $\hbar \omega_{-1}$ versus $\tau_{21}$ for $\rho=$ $6.4 \times 10^{9} \mathrm{~cm}^{-2}$ for a series of different central laser frequencies. The curve offsets are a real effect due to intraband renormalization of the dc field.

the right hand path, the exciton additionally interacts with the spatially uniform field, $E_{0}^{\text {intra }}(t)$, absorbing a photon of energy $\hbar \omega_{\text {intra }}^{0} \simeq \pm \hbar \omega_{\mathrm{B}}$ before reaching the final state $\left(\mu, 2 \boldsymbol{\kappa}_{2}-\boldsymbol{\kappa}_{1}\right)$.

To understand how this two-path interference leads to peak oscillations, we consider a simplified model wherein we assume that we have an intraband field, 
$E_{k}^{\text {intra }}(t)$, given by the second-order response. We then determine the expression for the DFWM interband response in the presence of this intraband field and the applied optical fields. The final expression is rather complicated. However, the basic result is very similar to what one would obtain in the calculation of the interband response for the structure where the two paths are: (1) direct linear absorption and (2) two-photon absorption, where the second photon is due to the component of the intraband field, $E_{0}^{\text {intra }}(t)$ at the Bloch frequency [6]. Using this analysis, and retaining only resonant terms, we obtain the expression, $P_{2 \boldsymbol{\kappa}_{2}-\boldsymbol{\kappa}_{1}}^{(\mathrm{L})}(\omega) \sim\left(\omega-\omega_{\mu}+\mathrm{i} / T_{\text {inter }}\right)^{-2}$, for the intraband polarization near $\omega=\omega_{\mu}$ due to the left-hand path and the expression, $P_{2 \kappa_{2}-\kappa_{1}}^{(\mathrm{R})}(\omega) \sim$ $P_{2 \boldsymbol{\kappa}_{2}-\boldsymbol{\kappa}_{1}}^{(\mathrm{L})}(\omega) E_{0}^{\mathrm{intra}}\left(\omega_{\mathrm{B}}\right) /\left(\omega-\omega_{\mu}+\mathrm{i} / T_{\text {inter }}\right)$, for the right-hand path polarization, where $E_{0}^{\mathrm{intra}(2)}\left(\omega_{\mathrm{B}}\right) \sim C \mathrm{e}^{-\mathrm{i} \omega_{\mathrm{B}} \tau_{21} / 2} \cos \left(\omega_{\mathrm{B}} \tau_{21} / 2\right)$ is the approximate expression for the amplitude of the intraband field at the Bloch frequency, and $C$ is a positive real constant. The oscillation of $E_{0}^{\text {intra }}\left(\omega_{\mathrm{B}}\right)$ with time delay is due to alternating constructive and destructive interference between the Bloch oscillating intraband polarizations created by the first and second pulses.

Using the above three expressions, we see that the SR-DFWM signal (which is proportional to the square of the total interband polarization) near the resonant peak at $\omega=\omega_{\mu}$ is given approximately by

$$
\begin{gathered}
I_{2 \boldsymbol{\kappa}_{2}-} \boldsymbol{\kappa}_{1}(\omega)=\frac{I_{0}}{\left[\left(\omega-\omega_{\mu}\right)^{2}+1 / T_{\text {inter }}^{2}\right]^{2}}\left\{1+\frac{C}{\left[\left(\omega-\omega_{\mu}\right)^{2}+1 / T_{\text {inter }}^{2}\right]}\right. \\
\left.\times\left[\left(\frac{C}{2}+\left(\omega-\omega_{\mu}\right)\right)\left(1+\cos \left(\omega_{\mathrm{B}} \tau_{21}\right)\right)+\frac{\sin \left(\omega_{\mathrm{B}} \tau_{21}\right)}{T_{\text {inter }}}\right]\right\} .
\end{gathered}
$$

Thus, for $\tau_{21}=\tau_{\mathrm{B}} / 2,3 \tau_{\mathrm{B}} / 2, \ldots$ the peak is at $\omega=\omega_{\mu}$, while for $\tau_{21}=0, \tau_{\mathrm{B}}, \ldots$, the peak is shifted maximally to higher frequencies. This is approximately what is seen in the results of the full calculation shown in Fig. 4. In addition, for intermediate delay times of $\tau_{21}=-\tau_{\mathrm{B}} / 4,3 \tau_{\mathrm{B}} / 4, \ldots$, a dip may appear in the spectra at $\omega=\omega_{\mu}$. Indeed, we observe such dips (or peak splitting) near $\tau_{21}=0.2 \mathrm{ps}$ and $0.6 \mathrm{ps}$ for $\omega_{\mathrm{c}}=\omega_{0}-1.72 \omega_{\mathrm{B}}$, as evidenced by the sudden changes in the peak position in Fig. 4.

Most of the features of the calculated peak oscillations agree with experiment: the phase, frequency, dependence on $\omega_{\mathrm{c}}$, and large amplitude at $\tau_{21}=0$, are all in general agreement $[9,10]$. However, the amplitude of the peak oscillation is considerably smaller than that obtained experimentally. For example, for $\omega_{\mathrm{c}}=\omega_{0}-2.27 \omega_{\mathrm{B}}$, and a density of $10^{10} \mathrm{~cm}^{-2}$, the calculated amplitude is roughly $0.6 \mathrm{meV}$, while the experimental amplitude is approximately $2.3 \mathrm{meV}$ [10]. This difference is likely caused by the screening of exciton-exciton interactions via incoherent carriers $[3,10]$. 


\section{Conclusions}

In summary, we applied the newly-developed infinite-order excitonic Bloch equations to calculate the $\mathrm{THz}$ emission and DFWM signals from a BSSL. The results show that the BSSL is a highly nonlinear system, with the interactions between the intraband and interband polarizations creating density-dependent effects that go well beyond third order in the optical field.

\section{Acknowledgments}

This work was supported in part by the Natural Sciences and Engineering Research Council of Canada, The European Commission Center of Excellence in Processing, Research, and Application of Advanced Materials (PRAMA), and the U.S. Office of Naval Research,

\section{References}

[1] V.M. Axt, S. Mukamel, Rev. Mod. Phys. 70, 145 (1998).

[2] M.M. Dignam, M. Hawton, Phys. Rev. B 67, 035329 (2003).

[3] M. Hawton, M.M. Dignam, Phys. Rev. Lett. 90, 267402 (2003).

[4] T. Meier, G. von Plessen, P. Thomas, S.W. Koch, Phys. Rev. Lett. 73, 902 (1994).

[5] V.M. Axt, G. Bartels, A. Stahl, Phys. Rev. Lett. 76, 2543 (1996).

[6] M.M. Dignam, Phys. Rev. B 59, 5770 (1999).

[7] L. Yang, B. Rosam, J.-M. Lachaine, K. Leo, M.M. Dignam, Phys. Rev. B 69, 165310 (2004).

[8] P.H. Bolivar, F. Wolter, A. Muller, H.G. Roskos, H. Kurz, K. Köhler, Phys. Rev. Lett. 78, 2232 (1997)

[9] V.G. Lyssenko, G. Valušis, F. Löser, T. Hasche, K. Leo, M.M. Dignam, K. Köhler, Phys. Rev. Lett. 79, 301 (1997).

[10] F. Löser, M.M. Dignam, Yu.A. Kosevich, K. Köhler, K. Leo, Phys. Rev. Lett. 85, 4763 (2000). 